\title{
Extracellular Onchocerca-derived small RNAs in host nodules and blood
}

Juan F Quintana ${ }^{1}$, Benjamin L Makepeace ${ }^{2}$, Simon A Babayan ${ }^{3}$, Alasdair Ivens ${ }^{1}$, Kenneth M Pfarr ${ }^{4}$, Mark Blaxter ${ }^{1}$, Alexander Debrah ${ }^{5}$, Samuel Wanji6, Henrietta F Ngangyung ${ }^{7}$, Germanus S Bah ${ }^{7}$, Vincent N Tanya ${ }^{8}$, David W Taylor ${ }^{2,9}$, Achim Hoerauf ${ }^{4}$ and Amy H Buck ${ }^{1 *}$

\begin{abstract}
Background: microRNAs (miRNAs), a class of short, non-coding RNA can be found in a highly stable, cell-free form in mammalian body fluids. Specific miRNAs are secreted by parasitic nematodes in exosomes and have been detected in the serum of murine and dog hosts infected with the filarial nematodes Litomosoides sigmodontis and Dirofilaria immitis, respectively. Here we identify extracellular, parasite-derived small RNAs associated with Onchocerca species infecting cattle and humans.

Methods: Small RNA libraries were prepared from total RNA extracted from the nodule fluid of cattle infected with Onchocerca ochengi as well as serum and plasma from humans infected with Onchocerca volvulus in Cameroon and Ghana. Parasite-derived miRNAs were identified based on the criteria that sequences unambiguously map to hairpin structures in Onchocerca genomes, do not align to the human genome and are not present in European control serum.

Results: A total of 62 mature miRNAs from 52 distinct pre-miRNA candidates were identified in nodule fluid from cattle infected with 0 . ochengi of which 59 are identical in the genome of the human parasite $O$. volvulus. Six of the extracellular miRNAs were also identified in sequencing analyses of serum and plasma from humans infected with $O$. volvulus. Based on sequencing analysis the abundance levels of the parasite miRNAs in serum or plasma range from 5 to 127 reads/per million total host miRNA reads identified, comparable to our previous analyses of Schistosoma mansoni and L. sigmodontis miRNAs in serum. All six of the O. volvulus miRNAs identified have orthologs in other filarial nematodes and four were identified in the serum of mice infected with L. sigmodontis.

Conclusions: We have identified parasite-derived miRNAs associated with onchocerciasis in cattle and humans. Our results confirm the conserved nature of RNA secretion by diverse nematodes. Additional species-specific small RNAs from 0 . volvulus may be present in serum based on the novel miRNA sequences identified in the nodule fluid. In our analyses comparison to European control serum illuminates the scope for false-positives, warranting caution in criteria that should be applied to identification of biomarkers of infection.
\end{abstract}

Keywords: microRNAs, Extracellular RNA, Filarial nematode, Onchocerciasis, Host-pathogen

\footnotetext{
* Correspondence: a.buck@ed.ac.uk

${ }^{1}$ Centre for Immunity, Infection and Evolution, Ashworth Laboratories,

University of Edinburgh, West Mains Road, Edinburgh, UK

Full list of author information is available at the end of the article
} 


\section{Background}

Small non-coding RNAs (sncRNAs) have emerged as important regulators of many processes in animals, from development to immunity. MicroRNAs (miRNAs) are the best characterized class of sncRNA which operate by guiding the RNA-induced silencing complex (RISC) to specific messenger RNAs (mRNAs) inside cells, where they inhibit translation and de-stabilize the targeted mRNAs [1]. In parasitic nematodes and flatworms, miRNAs have been shown to have core roles in the physiology of development, differentiation and homeostasis and potentially drug resistance [2]. Studies in the last 7 years have demonstrated that miRNAs can also exist in a cell-free form in extracellular fluids, where they may play endocrine signalling roles, reviewed in [3]. For parasitic species, interacting with this signalling system offers another potential mechanism of host manipulation. We and others have identified miRNAs from nematodes and trematodes in the serum of infected animals [4-6] and initial studies with $S$. mansoni demonstrated the utility of these molecules in distinguishing uninfected and infected humans [4]. The exact origin of these circulating parasite RNAs is unknown, but proteomic analysis of Dicrocoelium dendriticum suggests RNAs are associated with exosomes secreted from the parasite surface [7] and it is possible that previously described microvesicles in schistosomes could also contain RNA [8]. Recently we showed that miRNAs are packaged within vesicles secreted by the gastrointestinal nematode Heligmosomoides polygyrus and that these derive from the intestine of the nematode. These secreted vesicles (and their cargoes) suppress Th2 innate immune responses in vivo and the miRNAs within them are transferred to host cells in vitro [9]. Homologues of some of the miRNAs secreted by $H$. polygyrus miRNAs were also found in serum of hosts infected with the filarial nematodes Litomosoides sigmodontis [9] and Dirofilaria immitis [5]. The miRNAs secreted by nematodes and platyhelminth parasites may be a new axis of host-parasite interaction. Here we characterize the extracellular, parasite-derived miRNAs associated with the important human disease onchocerciasis.

Filarial infections currently affect over 150 million people in tropical and subtropical regions [10], with Onchocerca volvulus accounting for approximately 30.4 million [11] of which more than 99\% occur in Africa. Onchocerciasis is characterised by skin disease, which can be very severe, and is also the second leading cause of infectious blindness. Onchocerca ochengi, a filarial parasite of cattle, is the closest relative of $O$. volvulus, with which it is sympatric, and shares several key features with the human parasite $[12,13]$. Specifically, O. ochengi induces the formation of onchocercomata with very similar histological structure to human nodules [14], and both O. ochengi and O. volvulus present comparable mating behaviour within the nodules and subsequent Mf production, leading to a patent infection over a similar timescale [12]. The phylogenetic closeness means that the two species have very similar genomes, and thus very closely related (sometimes identical) antigens are present in both. There is evidence of cross-protection [15]. Therefore, O. ochengi represents the most relevant experimental model to understand the crosstalk between the parasite and the host in the context of onchocerciasis.

Since 1989, ivermectin has been used in mass drug administration (MDA) programmes to control onchocerciasis in Africa and Latin America. Following the success of the Onchocerciasis Elimination Program for the Americas, which has used MDA of ivermectin alone to abrogate transmission in most endemic foci, the goal of the African Programme for Onchocerciasis Control (APOC; which covers a vastly greater area) has shifted from control to eradication [13]. However, major challenges to this endeavour remain, such as the emergence of ivermectin resistance [16], the potential for severe adverse reactions to ivermectin in loiasis-endemic areas [17], and significant limitations in the accurate and rapid diagnosis of infection [18]. Currently, diagnosis relies on identification of microfilariae in skin snips, which are laborious and notoriously insensitive; additionally, this procedure can cause considerable discomfort. The availability of immunoassays such as the Ov16 serological test [19] has greatly enhanced the ability to detect residual transmission or the re-emergence of infection by using young children as "sentinels"; however, the longevity of immune responses in onchocerciasis renders this assay unsuitable as a tool to confirm elimination of infection from adults [20].

Detection of parasite DNA in a wide variety of bodily fluids by either polymerase chain reaction (PCR) or highthroughput deep sequencing has proven to be successful in the diagnosis of infections caused by $S$. mansoni, gastrointestinal parasitic nematodes [21] and Leishmania [22], among others. DNA-based tests thus represent an alternative diagnostic platform to conventional parasitological or antigen-based assays. sncRNAs are another class of diagnostic biomarker that can be amplified and are detectable by qRT-PCR. miRNAs are generally $\sim 22 \mathrm{nt}$ in length and have been detected outside of cells in many mammalian body fluids indicating that these molecules can be rendered highly stable and protected against extreme conditions (i.e. low $\mathrm{pH}$, degradation by extracellular RNases, etc.) [23]. The functional significance of their extracellular existence is still elusive $[3,23]$ but they have been shown to act locally in cell-to-cell communication in mammalian systems [3] and can also be moved from parasite to host via exosomes [9].

Here we report the detection and identification of Onchocerca spp. miRNAs from bovine nodular fluid ex vivo and the detection of a subset of these molecules in the serum 
and plasma of human onchocerciasis patients from Ghana and Cameroon. Several of these miRNAs are orthologs of (and in some cases have identical sequence to) those previously identified in serum of mice infected with $L$. sigmodontis as well as miRNAs secreted by $H$. polygyrus in vitro. Our findings indicate that miRNA secretion by nematodes is conserved in Onchocerca species.

\section{Methods}

\section{O. ochengi nodule fluid}

Bovine skins containing numerous $O$. ochengi onchocercomata were obtained from Ngaoundéré abattoir, Adamawa Region, Cameroon [24]. Freshly excised nodules were screened visually, and discarded if hard or discoloured, which are signs of calcification. Nodules were rinsed in PBS, dried thoroughly, and pricked with a 21G hypodermic needle. The nodules were gently squeezed, and the expressed fluid $(\sim 0.5 \mu \mathrm{l})$ collected with a micropipette, pooling from $>10$ nodules per biological replicate. The fluid was spun at $500 \mathrm{~g}$ for $5 \mathrm{~min}$ to pellet any cellular material or Mf, and the supernatant was stored at $-80^{\circ} \mathrm{C}$. The samples were shipped to the UK on dry ice and remained frozen prior to analysis.

\section{Human serum samples}

Archived human plasma from O. volvulus infected and uninfected volunteers was collected as part of a European Union Seventh Framework Programme Research grant, contract 131242 "Enhanced Protective Immunity Against Filariasis (EPIAF)", (http://cordis.europa.eu/project/rcn/94066_en.html). Infected individuals were those with palpable nodules and microfilaridermia by skin snip. Uninfected individuals were defined as persons with no palpable nodules and microfilariae negative skin snips. Serum or EDTA plasma was collected as previously described [25].

\section{Ethics statement}

The Committee on Human Research Publication and Ethics at the University of Science and Technology in Kumasi, Ghana, and the Ethics Committee at the University of Bonn, Germany approved the use of archived plasma samples. Collection of sera from onchocerciasis patients in Cameroon was approved by the Cameroon Ethics Committee and the Ministry of Public Health as part of the EU FP7 contract 131242 (EPIAF) and in compliance with the Helsinki declaration on the use of humans in biomedical research. Prior to recruitment, the nature and objectives of the study were explained to potential participants and those who agreed to take part in the study signed a consent form while an assent was obtained from parents or guardians of children who were enrolled in the study. Participation was voluntary.

\section{RNA extraction and library preparation}

RNA was extracted from $20 \mu \mathrm{L}$ of pooled nodule fluids from cattle (O. ochengi infection), $200 \mu \mathrm{L}$ of serum pooled from 12 infected individuals in Cameroon (pooled prior to RNA extraction), or pooled from equal volumes of RNA extracted from 13 infected or 13 uninfected individuals in Ghana (total equivalent of $50 \mu \mathrm{L}$ plasma). Samples were shipped in liquid nitrogen and stored at $-80^{\circ} \mathrm{C}$ for $3-4$ years (Cameroon samples) or 5 years (Ghana samples). The serum was thawed on ice and serum or plasma was spun down at $16,000 \mathrm{~g}$ for $5 \mathrm{~min}$ at $4^{\circ} \mathrm{C}$ to remove any additional cell debris. The cleared serum was then transferred to a new $2 \mathrm{~mL}$ Eppendorf tube and RNA extracted using the miRCURY RNA isolation kit for Biofluids (Exiqon) according to manufacturers' protocols. In both cases, RNA was eluted in $50 \mu \mathrm{L}$ of $0.1 \mathrm{mM}$ EDTA. RNA was stored at $-20^{\circ} \mathrm{C}$ prior to further analysis. The relative small RNA content from these samples was determined with $1 \mu \mathrm{L}$ of total RNA on a Bioanalyzer small RNA chip (Agilent).

Before proceeding with small RNA library preparation from serum or plasma RNA, samples were cleaned up as in [26]. Briefly, $50 \mu \mathrm{L}$ of eluted RNA was diluted to $100 \mu \mathrm{L}$ with Nuclease-free MiliQ water followed by addition of $1 \mu \mathrm{L}$ glycoblue $15 \mathrm{mg} / \mathrm{ml}$ (Life technologies), $60 \mu \mathrm{L}$ of Sodium acetate $3 \mathrm{M} \mathrm{pH} 5.2$ (AppliChem) and $500 \mu \mathrm{L}$ of ethanol $100 \%$. The RNA was precipitated for $30 \mathrm{~min}$ at $-80^{\circ} \mathrm{C}$ then spun at $16,000 \mathrm{~g}$ for $30 \mathrm{~min}$ at $4^{\circ} \mathrm{C}$ and washed twice with $75 \%$ Ethanol. The pellets were air-dried at room temperature for 15 minutes and resuspended in $8 \mu \mathrm{L}$ of $0.1 \mathrm{mM}$ EDTA pH 8.0.

For the analysis of small RNA content in these nodule fluids or human serum and plasma, libraries were prepared from total RNA using the Illumina TruSeq small RNA Preparation kit, according to the manufacturers' protocol, and using 1:10 dilution of adapters. PCR products of the expected molecular weight (140-160 bp) were size selected and sequenced on an IlluminaHiSeq2500 instrument using v3 reagents in Edinburgh Genomics (http://genomics.ed.ac.uk/).

\section{Bioinformatic analysis}

All libraries were analysed by first clipping the 3' sRNA adapter using cut adapt [27], searching for at least a 6 base match to the adapter sequence. For analysis of small RNAs, only sequences that contained the adapter, were $>16 \mathrm{nt}$ in length, and were present at $\geq 2$ copies were retained for further analysis. Sequences were analyzed for alignment to bovine (ftp://ftp.ensembl.org/pub/release-71/fasta/bos_taurus/ dna/Bos_taurus.UMD3.1.71.dna.toplevel.fa.gz), human (version hg19), O.ochengi (version 1.1; from http://onchocerca. nematod.es; unpublished genome sequence from $\mathrm{M}$. Blaxter, B. Makepeace and colleagues) or O. volvulus (ftp://ftp. sanger.ac.uk/pub/project/pathogens/Onchocerca/volvulus/ 
OVOC.V3.fa) using bowtie [28], requiring perfect matches along the full length of the sequence. Sequences were analyzed for known classes of RNA based on Rfam [29] (version 11, obtained from ftp://ftp.sanger.ac.uk/pub/ databases/Rfam/11.0/). The best hit with at most two mismatches was used to classify the reads. Analysis of miRNA content was carried out using miRdeep2 [30] with the following settings: 1 ) reads map perfectly to the genome, 2) cut off -v 1,3) employing the "-s option" using all mature sequences from mirbase (version 20) [31], 4) only read lengths (18 to $30 \mathrm{nt}$ ) were analyzed. Folding analyses of the novel pre-miRNAs detected in nodule fluids were carried out using the RNAfold Vienna package with default settings [32].

\section{Results}

O. ochengi small RNAs are present in bovine nodule fluid

RNA from the fluid of nodules of cattle infected with $O$. ochengi was analysed for small RNA content using the Agilent Bioanalyzer. Two populations were observed between 20-30 nt and 50-70 nt (Figure 1), similar to the small RNA profile previously observed for the in vitro secretion products of the gastrointestinal nematode $H$. polygyrus [9]. Small RNA libraries were prepared and sequenced to identify the small RNAs between 17 and $40 \mathrm{nt}$ and analysed as previously described [4]. Importantly, to avoid analysis of sequencing artefacts, reads that were present in $<2$ copies were discarded and according to default criteria of miRdeep2 alignments only reads $>16 \mathrm{nt}$ were analysed. To further increase confidence in our assignments, we required that reads contained the 3 ' adapter and aligned along their full length to the bovine or $O$. ochengi draft genomes. Since some small RNA sequences can be post-transcriptionally edited this method may miss true positives. Following these criteria, a total of 15,043,191 reads were analysed of which 11,905,898 aligned to the bovine genome and 151,332 aligned to the O. ochengi genome (Table 1). A total of 6,301 reads that could be equivalently aligned to both genomes were not included in the analysis since their origin could not be determined.

The small RNA content was initially classified based on sequence identity (assessed using BLAST [33]) to known RNA classes in Rfam and revealed a predominance of tRNA fragments (Table 1), as previously observed in other extracellular fluids [4,34]. Identification of miRNAs was carried out using miRdeep2 which not only identifies matches to miRNAs already present in miRbase but also identifies novel miRNAs based on the ability of the reads to map to potential hairpins in the genome [30]. From this analysis a total of 62 mature miRNAs were identified including 23 that are identical to previously described nematode miRNAs (primarily Ascaris suum and Brugia malayi), 18 that are only identical in their seed sites (nucleotides 2 to 8 ) to other nematode miRNAs, and 21 of which did not

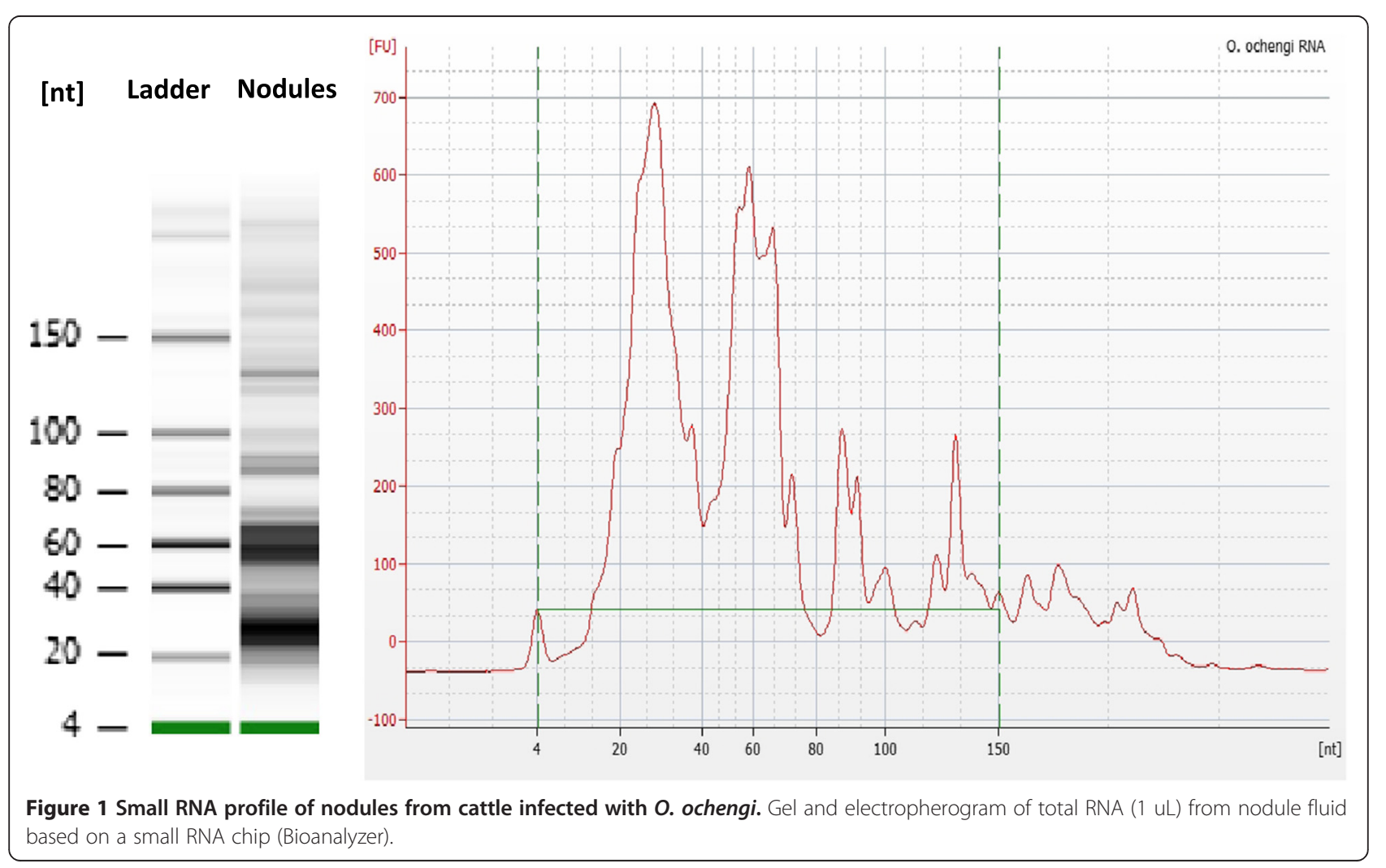


Table 1 Bovine and O.ochengi small RNA classification in nodules

\begin{tabular}{ll}
\hline Trimmed reads & $\mathbf{1 5 0 2 2 2 7 8}$ \\
\hline Bovine genome match & $\mathbf{1 1 9 1 2 1 9 9}$ \\
\hline Unambigious & 11905898 \\
rRNA & 20836 \\
tRNA & 11335037 \\
Y RNA & 51307 \\
Other Rfam & 25716 \\
miRNA & 382791 \\
O. ochengi genome match & $\mathbf{1 5 7 6 3 3}$ \\
\hline Unambigious & 151332 \\
rRNA & 2616 \\
tRNA & 120733 \\
Y RNA & 0 \\
Other Rfam & 2344 \\
miRNA & 11455 \\
\hline
\end{tabular}

share homology in their seed sites (Additional file 1: Table S1). From these analyses we identify 16 novel pre-miRNA candidates that are not homologs of other known nematode pre-miRNAs. Six of these have reads mapping specifically to both arms of the hairpin with 3' overhangs (Figure 2) and we therefore assign confidence to their classification as Dicer-derived miRNAs. To determine whether these small RNAs are conserved in the closely related human parasite $O$. volvulus, miRdeep 2 analysis of the reads sequenced in the $O$. ochengi nodule material was carried out using the $O$. volvulus draft genome as the mapping substrate, allowing for up to 2 mismatches. All sequences aligned perfectly and derived from hairpins, apart from three miRNAs (Ooc-novel-3-3p, Ooc-novel-15, Ooc-miR49) where a $1 \mathrm{nt}$ mismatch was present (Additional file 1 : Table S1).

\section{Six parasite-derived miRNAs are detected in serum or plasma of humans infected with 0 . volvulus}

To date very little is known about the factors that dictate the stability of extracellular RNA in fluids or whether and how these traffic within the body. The nodules are highly vascularized [35] and provide a direct route to the blood system for parasite-derived molecules. To determine whether $O$. volvulus-derived miRNAs are present in serum and plasma we carried out two parallel analyses of samples pooled from infected humans obtained from two geographically relevant regions in Africa, Cameroon [36] and Ghana [37], and compared these to pooled endemic or European controls. At present there is no standardized way to assess the integrity or quality of RNA extracted from serum since the intact large ribosomal RNAs (rRNAs) are not present. However we observed a distinct population of small RNA that is $20-30$ nt in length based on Bioanalyzer analyses. This population was more prominent when using the Exiqon biofluids extraction kit compared to the Qiagen miRNA easy kit (Additional file 2: Figure S1). Small RNA sequencing libraries were prepared, sequenced and analysed as above. A total of 10-25 million reads were analysed per sample, of which $39-51 \%$ mapped to the human genome and

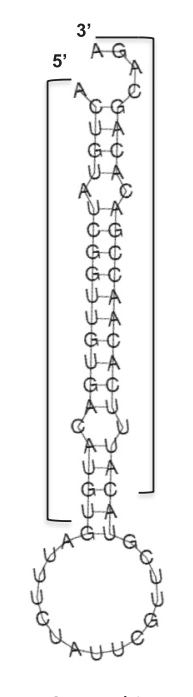

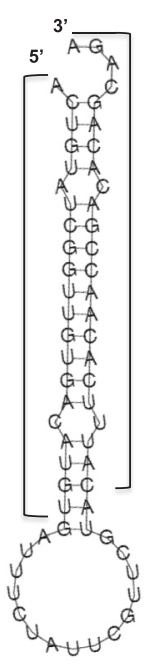

Ooc-novel-2

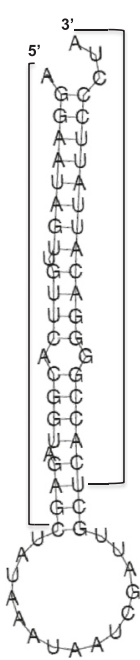

Ooc-novel-3

$\Delta \mathrm{G}=-23.90 \mathrm{kcal} / \mathrm{mo})$

Figure 2 Novel O.ochengi pre-miRNAs identified in bovine nodule fluid. Predicted secondary structure of the novel pre-miRNAs identified by presence of reads mapping to both $5 p$ and $3 p$ arms (indicated in brackets). The minimum free energies (MFE) are indicated according to prediction by RNAfold. 
1.0-1.5\% mapped to the draft O. volvulus genome. The majority of the reads from human serum that mapped to O. volvulus were identified as rRNA fragments, but these were detected at comparable levels in European control and infected samples. It is possible these are of human origin but are edited or that they derive from other organisms or dietary sources as shown in several studies [38,39]. Similarly the source of many reads that map to O. volvulus genome and are annotated as tRNA fragments [34] cannot be reliably assigned (Table 2).

Here we focus on the miRNAs detected in the serum from humans testing positive for $O$. volvulus that can be assigned a nematode origin. From the combined datasets we identify a total of six O. volvulus miRNAs. The nematode origin of these is evident from a number of criteria: 1) they map perfectly to regions that fold into hairpin structures within the $O$. volvulus genome; 2 ) they are not other classes of sncRNA and 3) they are not present in the sera of European controls. Of the six miRNAs identified, all were identical to the $O$. ochengi miRNAs found in nodules (Table 3). Two of the miRNAs, miR-71 and lin-4, are detected in infected samples from both Ghana and Cameroon but neither endemic nor European controls. Two are detected only in the infected pooled sample from Ghana (miR-100a, miR-87) and two of these are present in infected and endemic control samples from Ghana (miR-100d, bantam-a). As the endemic control sample was a pool of 13 individuals, it is possible that these miRNAs could derive from an individual misdiagnosed as negative for onchocerciasis or co-infected with other co-endemic nematode parasites in these regions. Of note, miR-92 and let-7 were detected in some of the libraries however the mature miRNAs are perfectly conserved between nematodes and mammals. There can be some heterogeneity in the terminal nucleotides of these miRNAs based on non-templated additions [40] (as is common for miRNAs) such that they could technically align better to parasite than host. The exact origin(s) of these miRNA cannot be inferred.

\section{Common and distinct circulating miRNA signatures in filarial infections}

We recently identified 16 miRNAs in the serum of mice infected with the filarial nematode $L$. sigmodontis and four of these are identical to the O. volvulus miRNAs detected in human serum (mir-71, two miR-100 members, and one bantam family member) and one is derived from the other arm of the hairpin of a O. volvulus miRNA (miR-87). A further seven of the L.sigmodontis miRNAs are identical to $O$. ochengi miRNAs found in the nodule fluid and three (miR-50-3p and Bantam-b,c) differ by $1 \mathrm{nt}$ outside of the seed region (Figure 3). Strikingly multiple miR-100 and bantam family members are present in the datasets. These also dominate the secretion product of the gastrointestinal nematode $H$. polygyrus [9]. The O. volvulus miR-100 and bantam miRNAs identified have distinct sequences outside of their seed regions from the miRNAs in H. polygyrus (Figure 4).

To compare relative quantities across the datasets and to qualify our limit of detection, we used total host miRNA read counts in the same samples as a normalizer. The individual parasite miRNAs are detected at a range of 26 to 12,863 per million bovine miRNA reads (O. ochengi nodule fluid), 5 to 127 per million human miRNA reads (serum/

Table 2 Small RNA classification in human serum and plasma from uninfected and infected individuals

\begin{tabular}{|c|c|c|c|c|}
\hline Human serum/plasma & $\begin{array}{l}\text { Uninfected serum } \\
\text { (European control) }\end{array}$ & $\begin{array}{l}\text { Infected serum } \\
\text { (Cameroon) }\end{array}$ & $\begin{array}{l}\text { Uninfected plasma } \\
\text { (Ghana) }\end{array}$ & $\begin{array}{l}\text { Infected plasma } \\
\text { (Ghana) }\end{array}$ \\
\hline Trimmed reads & 25519512 & 23734119 & 24992446 & 10015190 \\
\hline Human genome match & 9998552 & 9331113 & 12936180 & 4791645 \\
\hline Unambiguous & 9937398 & 9102817 & 12846400 & 4742963 \\
\hline rRNA & 15854 & 26512 & 13022 & 6744 \\
\hline tRNA & 2568803 & 82382 & 6442858 & 1724586 \\
\hline Y RNA & 1149988 & 2408124 & 1004222 & 476464 \\
\hline Other Rfam & 198310 & 33516 & 8497 & 3791 \\
\hline miRNA & 5589367 & 5924748 & 5266797 & 2472450 \\
\hline O. volvulus genome match & 304991 & 583693 & 328590 & 157005 \\
\hline Unambiguous & 243837 & 355397 & 238810 & 108323 \\
\hline rRNA & 140174 & 108351 & 132900 & 49797 \\
\hline tRNA & 358 & 400 & 1884 & 1929 \\
\hline Y RNA & 50 & 0 & 0 & 0 \\
\hline Other Rfam & 2434 & 2712 & 3013 & 688 \\
\hline miRNA & 0 & 75 & 344 & 743 \\
\hline
\end{tabular}


Table 3 Read numbers of nematode-derived miRNAs detected in serum or plasma from individuals who tested positive for 0 . volvulus

\begin{tabular}{|c|c|c|c|c|c|c|}
\hline miRNA & RNA sequence & Precursor coordinates & $\begin{array}{l}\text { Uninfected serum } \\
\text { (European control) }\end{array}$ & $\begin{array}{l}\text { Infected serum } \\
\text { (Cameroon) }\end{array}$ & $\begin{array}{l}\text { Uninfected } \\
\text { serum (Ghana) }\end{array}$ & $\begin{array}{l}\text { Infected } \\
\text { serum (Ghana) }\end{array}$ \\
\hline miR-71 & UGAAAGACAUGGGUAGUGAGAC[G] $^{1}$ & OVOC.OM1b:9907432..9907494:+ & 0 & 43 & 0 & 98 \\
\hline lin-4 & UCCCUGAGACCUCUGCUGCGA & OVOC.OM4:5453650..5453708:- & 0 & 32 & 0 & 73 \\
\hline miR-100d & AACCCGUAGUUUCGAACAUGUGU & OVOC.OM1a:1762729..1762789:- & 0 & 0 & 121 & 314 \\
\hline miR-87-3p & GUGAGCAAAGUUUCAGGUGUUC & OVOC.OM2:17655901..17655965:- & 0 & 0 & 0 & 85 \\
\hline miR-100a & UACCCGUAGCUCCGAAUAUGUGU & OVOC.OM1a:1763611..1763671:- & 0 & 0 & 0 & 102 \\
\hline bantam-a & UGAGAUCAUUGUGAAAGCUAUU & OVOC.OM2:1211194..1211257:- & 0 & 0 & 223 & 71 \\
\hline
\end{tabular}

${ }^{1}$ Brackets indicate heterogeneity in the 3 ' terminal nucleotide between datasets.

plasma from $O$. volvulus positive individuals) and 2 to 367 reads per million mouse miRNAs (serum from L. sigmodontis infected mice) as shown in Table 4. We anticipate these relative proportions will vary depending on the intensity of infection. Importantly, this provides a baseline for comparing relative levels of parasite miRNAs between studies and understanding the limits of detection in each study.

A recent analysis of serum from humans infected with $O$. volvulus using deep sequencing reported 20 putative $O$. volvulus miRNAs that have no overlap with those we identify here [5]. Thirteen of the reported sequences were $<17$ nt long, or detected in only one read and thus are not analysed by our criteria. Of the seven additional sequences three of these perfectly align to human ribosomal RNA and two of these (PC-5p-31768_12, PC-3p-
46055_7) are found as a part of longer sequences in European control serum. One of the sequences, let-7, is also found in our datasets but we do not confidently assign this to nematode origin given its conservation in the mammalian host.

\section{Discussion}

The discovery that RNA is secreted by nematodes opens up many avenues for further investigation into their functional properties and diagnostic utility. Here we report that small noncoding RNAs derived from Onchocerca spp. are present in host tissues, both at a concentrated site of infection (nodule fluid) and in the circulatory system (serum/plasma) of their hosts. Six O. volvulus miRNAs were identified in human plasma, all of which are identical to those found in $O$. ochengi nodule fluid, and four of

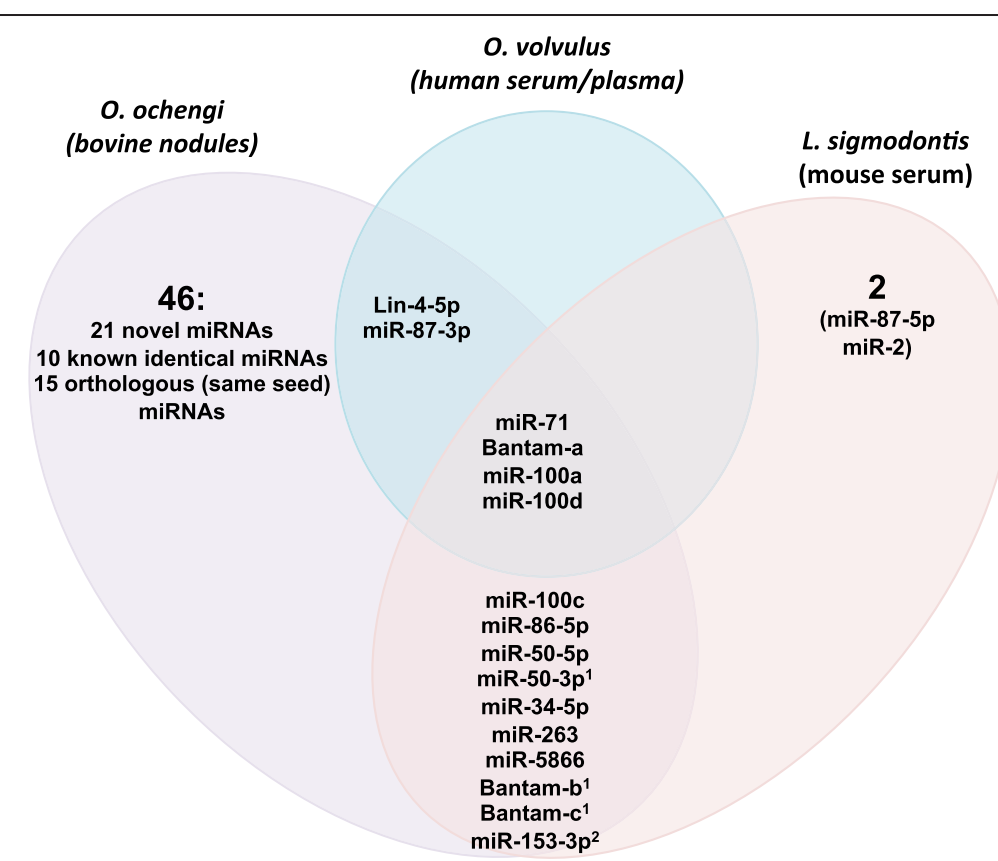

Figure 3 Venn diagram depicting overlap in extracellular miRNAs identified in filarial infections. Overlapping mature miRNAs sequences identified in cattle nodule fluids (O. ochengi), serum/plasma from infected patients in Cameroon and Ghana (O. volvulus) and infected mouse serum (L. sigmodontis, as previously reported [9]). 'These miRNAs differ by $1 \mathrm{nt}$ outside of the seed region in L. sigmodontis and O. ochengi. ${ }^{2}$ miR-153-3p is identical to mammalian miR-153-3p from nucleotides 1-21. 


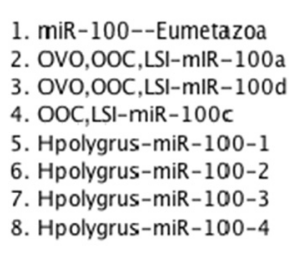

1. S.mansoni

2. OVO,OOC,LSI-bantam-a

3. OOC-bantam-b

4. LSI-bantam-b

5. OOC-bantam-c

6. LSI-bantam-c

7. Hpolygyrus-bantam-1

8. Hpolygyrus-bantam-2

9. Hpolygyrus-bantam-3

10. Hpolygyrus-bantam-4

11. Hpolygyrus-bantam-5
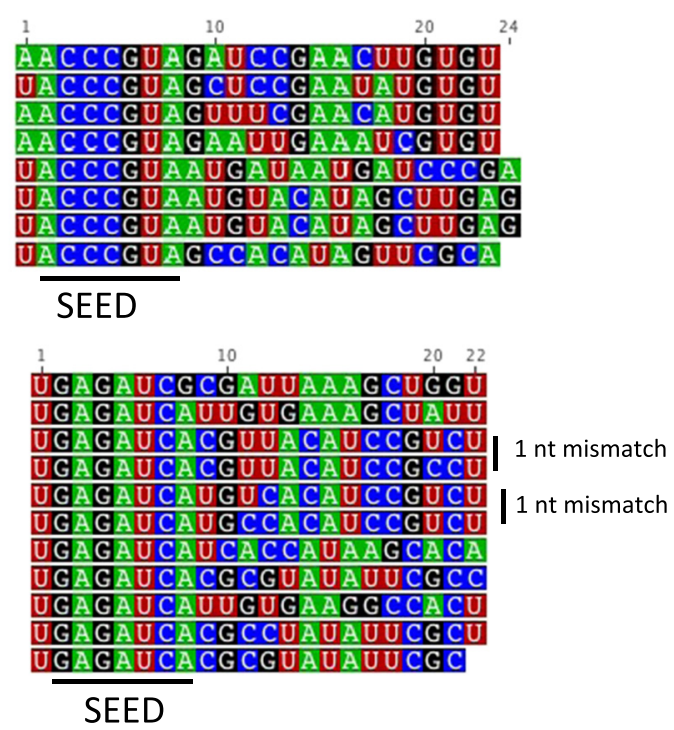

Figure 4 Sequences of extracellular miR-100 and bantam family members in nematode parasites. The conserved miR-100 sequence (Eumetazoa) is shown in relation to the nematode family members identified in these datasets: O. volvulus (OVO), O. ochengi (OOC), L. sigmodontis (LSI) or H. polygyrus (Hpolygyrus). The S. mansoni bantam sequence is taken from Hoy et al., PLos NTDS 2014 [4]. The naming of family members is arbitrary.

which are also identical to those found in serum of mice infected with the related filarial nematode $L$. sigmodontis (Figure 3). This suggests extensive overlap in the identity of extracellular parasite-derived miRNAs in filarial infections and gives confidence in the conserved nature of RNA secretion among these pathogens. This is further supported by a report published while this manuscript was in preparation which identified miRNA candidates of potential nematode origin in the plasma of baboons infected with Loa loa and the plasma of an O. ochengiinfected cow [41]: 4 of the 6 miRNAs that we identify in O. volvulus-infected humans are among the 22 miRNA candidates found in Loa loa-infected baboons and 2 of the 62 O. ochengi miRNAs in nodules are among the 10 candidates found in bovine plasma (Additional file 1: Table S1).

A common feature in all the infections is the presence of miR-71, bantam family and miR-100 family miRNAs (where family is defined based on identical seed sequences, nucleotides 2-8). We previously identified 5 miR-100 family members within the top 20 most abundant miRNAs secreted by $H$. polygyrus [9]. The factors dictating the expansion of this miRNA family are not known; miR-100 is

Table 4 Relative abundance of nematode miRNA in fluids in comparison to host miRNAs (reads per million)

\begin{tabular}{|c|c|c|c|c|c|}
\hline miRNA & Sequence & O. ochengi & O. volvulus (Ghana) & O. volvulus (Cameroon) & L. sigmodontis \\
\hline Ooc-miR-71 & UGAAAGACAUGGGUAGUGAGAC[G] ${ }^{3}$ & 117.6 & 39.6 & 7.3 & 25.1 \\
\hline Ooc-lin-4 & UCCCUGAGACCUCUGCUGCGA & 1355.8 & 29.5 & 5.4 & $\mathrm{ND}(<0.8)^{2}$ \\
\hline Ooc-miR-100d ${ }^{1}$ & AACCCGUAGUUUCGAACAUGUGU & 86.2 & 127.0 & $\mathrm{ND}(<0.3)^{2}$ & 31.4 \\
\hline Ooc-miR-87-3p & GUGAGCAAAGUUUCAGGUGUUC & 26.1 & 34.4 & $\mathrm{ND}(<0.3)^{2}$ & $\mathrm{ND}(<0.8)^{2}$ \\
\hline Ooc-miR-100a & UACCCGUAGCUCCGAAUAUGUGU & 979.6 & 41.3 & $\mathrm{ND}(<0.3)^{2}$ & 376.0 \\
\hline Ooc-bantam-a ${ }^{1}$ & UGAGAUCAUUGUGAAAGCUAUU & 12863.4 & 28.7 & $\mathrm{ND}(<0.3)^{2}$ & 35.3 \\
\hline Ooc-miR-86 & UAAGUGAAUGCUUUGCCACAGUCU & 65.3 & $\mathrm{ND}(<0.8)^{2}$ & $\mathrm{ND}(<0.3)^{2}$ & 44.7 \\
\hline Ooc-miR-263/183 & AAUGGCACUAGAUGAAUUCACGG & 44.4 & $\mathrm{ND}(<0.8)^{2}$ & $\mathrm{ND}(<0.3)^{2}$ & 5.5 \\
\hline Ooc-miR-50-5p & UGAUAUGUCUGAUAUUCUUGGGUU & 31.3 & $\mathrm{ND}(<0.8)^{2}$ & $\mathrm{ND}(<0.3)^{2}$ & 7.9 \\
\hline Ooc-miR-34 & UGGCAGUGUGGUUAGCUGGUUGU & 70.5 & $\mathrm{ND}(<0.8)^{2}$ & $\mathrm{ND}(<0.3)^{2}$ & 6.3 \\
\hline \multirow[t]{2}{*}{ Ooc-miR-5866 } & UUACCAUGUUGAUCGAUCUCC[A] ${ }^{3}$ & 70.5 & $\mathrm{ND}(<0.8)^{2}$ & $\mathrm{ND}(<0.3)^{2}$ & 1.60 \\
\hline & Total host miRNAs & 382791 & 2472450 & 5924748 & 1273839 \\
\hline
\end{tabular}

${ }^{1}$ Also found in endemic Ghanaian controls.

${ }^{2} \mathrm{ND}=$ not detected; the limit of detection is shown in (), based on the number of total host miRNAs sequenced and assuming 2 reads are required to identify a parasite sequence.

${ }^{3}$ Brackets indicate heterogeneity in the 3 ' terminal nucleotide between datasets. 
one of the oldest miRNAs, having evolved in the last common ancestor of Eumetazoa (the highly conserved sequence is noted in Figure 4 and is identical across parasitic nematodes and all of their mammalian hosts). This family has expanded in some animal lineages: in C. elegans it is referred to as the miR-51 family and is redundantly required for embryonic development [42] and also involved in developmental timing and buccal cavity formation $[42,43]$. Why members of this family are secreted by parasitic nematodes is unknown and raises interesting questions regarding whether these would interact with host targets. From a diagnostic standpoint it is worth noting that the sequences outside the seed region differ between the filarial nematodes and $H$. polygyrus (Figure 4). We also identify bantam family members in serum of both L. sigmodontis and O. volvulus infected hosts. Two of the bantam family members found in the serum of $L$. sigmodontis infected mice have a $1 \mathrm{nt}$ mismatch to the family members in O. ochengi and O. volvulus (Figure 4). One of the bantam members identified here appears conserved and secreted in all Clade III nematodes. We identified this miRNA in O. ochengi nodule fluid, serum from O. volvulus-infected individuals and serum from mice infected with $L$. sigmodontis. However, this miRNA sequence was also present in endemic controls from the Ghana cohort. This may represent a false negative individual or may occur if another parasite in one or more of the control individuals also secretes bantam orthologs. According to miRbase, this sequence is specific to filarial nematodes and is not present in the Clade $\mathrm{V}$ nematodes (Rhabditida; strongyles including $H$. polygyrus, free-living rhabditids and relatives). Interestingly, the secretion of bantam family members also occurs in trematodes; we previously identified a bantam family member (distinct in sequence from those identified here, Figure 4) in the serum of mice and humans infected with $S$. mansoni [44], and demonstrated its utility as a biomarker for schistosomiasis [4].

A key criterion in our analysis is the requirement that the annotated nematode miRNAs do not have a match in the host genome. A recent study reported putative O. volvulus miRNAs in human serum [5], some of which we annotate here as human ribosomal RNAs. This does not rule out that human sequences could also serve as a marker of infection, but it will be imperative to compare serum from uninfected individuals to avoid false positives and to build a better context for when and why these sequences can be detected.

We previously identified miRNAs derived from $L$. sigmodontis in mouse serum during patent infection [9]. However using the same library preparation methodology and sequencing at the same depth of coverage we did not detect miRNAs derived from the gastrointestinal nematode $H$. polygyrus in the serum of infected mice (at day 14, when the adult worms reside in the small intestine). Since the adult $H$. polygyrus worms secrete miRNAs within exosomes in vitro [9] it seems likely that lack of detection in serum relates to the localization of the parasite in the host. In support of this a recent report identified 245 putative parasite miRNAs in the serum of dogs infected with the heartworm $D$. immitis [5]. Individuals infected with this species of filaria would be expected to have a higher concentration of circulating parasitederived miRNAs (relative to other filariae) due to the presence of both $\mathrm{Mf}$ in the blood and adult worms in the pulmonary artery and heart.

As observed in the L. sigmondontis dataset the O. volvulus miRNAs are much less abundant than host miRNAs in serum or plasma: in the Ghana samples we mapped 743 reads to 6 different nematode-derived miRNAs, compared to approx. 2.5 million human miRNAs reads in the same library. Low-abundance is a challenge when detecting any type of parasite-derived molecule in host fluid. An advantage of the miRNAs is that these can be amplified by PCR prior to detection. Nonetheless, methods for enriching parasite material are likely to be advantageous in terms of maximizing the specificity and sensitivity of detection. In this regard, we have shown nematode miRNAs are secreted within extracellular vesicles in vitro [9]. Further work is required to understand whether parasite and host miRNAs exist in similar or distinct complexes in host fluids, and/or whether these can be further purified prior to RNA extraction to reduce the scope for crosscontamination.

The biology and dynamics of secreted miRNAs are thus very open topics. Nothing is known at present about the extent to which different parasite life stages secrete miRNAs or what their half-lives are in host tissues including blood. We anticipate that the diagnostic utility of these molecules will also depend on the degree to which RNA secretion is regulated, the mechanism by which the small RNAs enter circulation and the stability of each RNA species in different fluids.

\section{Conclusions}

We have identified a total of 62 miRNAs derived from $O$. ochengi in bovine nodule fluids, including miRNAs that are perfectly conserved in other filarial nematodes and some that do not have homology to other nematodes. Six of the conserved miRNAs are present in serum or plasma from humans testing positive for O. volvulus in Cameroon and Ghana. Four of these are also identical to those found in the serum of mice infected with L. sigmodontis including miR-100 and bantam family members. These findings support the conserved nature of RNA secretion by nematode parasites and identify miRNAs as a new potential biomarker for filarial infections that could 
significantly improve the diagnostic outlook for these neglected conditions. Further studies investigating exactly which parasite life stage(s) secrets such miRNAs, their stability, half-lives and localization within the host will drive forward the applications of parasite-specific miRNAs as biomarkers for onchocerciasis.

\section{Additional files}

Additional file 1: Table S1. miRNA candidates found in O.ochengi nodules and comparison to Loa loa and O.ochengi miRNA candidates reported in Tritten et al., Molecular \& Biochemical Parasitology 2014 [41].

Additional file 2: Figure S1. Small RNA profile of human serum comparing two different extraction kits. Gel of total RNA (1 uL) extracted from three replicates of human European control serum using two different kits: miRNeasy serum/plasma kit (Qiagen) and miRCURY RNA Isolation kit Biofluids (Exiqon) based on small RNA chip (Bioanalyzer).

\section{Competing interests}

The authors declare that they have no competing interests.

The data discussed in this publication have been deposited in NCBI's Gene Expression Omnibus (Barrett et al. 2013) and are accessible through GEO Series accession number GSE63933 (http://www.ncbi.nlm.nih.gov/geo/query/ acc.cgi?acc $=$ GSE63933)

\section{Authors' contributions}

JFQ performed experiments, co-designed studies and co-wrote the manuscript with AHB; BM, SAB, DWT, KMP and AH coordinated accessibility of material, edited manuscript and co-conceived project with AHB; Al, JFQ and AHB analysed the data, MB provided genome data, advice and edited manuscript, AD, SW, HFN, GSB, VNT provided crucial sample material. AHB wrote paper with $\mathrm{JFQ}$ and supervised the work. All authors read and approved the final version of the manuscript.

\section{Acknowledgements}

Funding was provided by the Institute for Medical Microbiology, Immunology and Parasitology, University Hospital of Bonn, Germany as part of a consortium grant funded by the Bill and Melinda Gates Foundation to the University Hospital of Bonn as well as a Wellcome Trust RCDF (WT097394AIA) to A. Buck. Funding for Cameroonian human sera and nodule fluid was provided by the 7th Framework Programme of the European Commission (project identifier HEALTH-F3-2010-242131). We thank Kat Gordon and Kashyap Chhatbar for helpful discussions on this work.

\section{Author details \\ ${ }^{1}$ Centre for Immunity, Infection and Evolution, Ashworth Laboratories, University of Edinburgh, West Mains Road, Edinburgh, UK. Institute of Infection and Global Health, University of Liverpool, Liverpool, Merseyside, UK. ${ }^{3}$ Institute of Biodiversity, Animal Health and Comparative Medicine, College of Medical, Veterinary and Life Sciences, University of Glasgow, Glasgow, UK. Institute of Medical Microbiology, Immunology and Parasitology, University Hospital of Bonn, Bonn, Germany. ${ }^{5}$ Kumasi Centre for Collaborative Research (KCCR) and Kwame Nkrumah University of Science and Technology, Kumasi, Ghana. ${ }^{6}$ Research Foundation in Tropical Diseases and Environment and University of Buea, Buea, Cameroon. Institut de Recherche Agricole pour le Développement, Regional Centre of Wakwa, Ngaoundéré, Cameroon. ${ }^{8}$ Cameroon Academy of Sciences, Yaoundé, Cameroon. ${ }^{9}$ Division of Pathway Medicine, School for Biomedical Sciences, University of Edinburgh, Little France, Edinburgh, UK.}

Received: 5 December 2014 Accepted: 12 January 2015

Published online: 27 January 2015

\section{References}

1. Fabian MR, Sonenberg N. The mechanics of miRNA-mediated gene silencing: a look under the hood of miRISC. Nat Struct Mol Biol. 2012;19:586-93.
2. Devaney $E$, Winter AD, Britton C. microRNAs: a role in drug resistance in parasitic nematodes? Trends Parasitol. 2010;26:428-33.

3. Turchinovich A, Samatov TR, Tonevitsky AG, Burwinkel B. Circulating miRNAs: cell-cell communication function? Front Genet. 2013;4(June):119.

4. Hoy AM, Lundie RJ, Ivens A, Quintana JF, Nausch N, Forster T, et al. Parasite-derived microRNAs in host serum as novel biomarkers of helminth infection. PLoS Negl Trop Dis. 2014;8:e2701.

5. Tritten L, Burkman E, Moorhead A, Satti M, Geary J, Mackenzie C, et al. Detection of circulating parasite-derived MicroRNAs in filarial infections. PLoS Negl Trop Dis. 2014;8:e2971.

6. Cheng G, Luo R, Hu C, Cao J, Jin Y. Deep sequencing-based identification of pathogen-specific microRNAs in the plasma of rabbits infected with Schistosoma japonicum. Parasitology. 2013;140:1751-61.

7. Bernal D, Trelis M, Montaner S, Cantalapiedra F, Galiano A, Hackenberg M, et al. Surface analysis of Dicrocoelium dendriticum. The molecular characterization of exosomes reveals the presence of miRNAs. J Proteomics. 2014;105:232-41.

8. Gobert GN, Stenzel DJ, McManus DP, Jones MK. The ultrastructural architecture of the adult Schistosoma japonicum tegument. Int J Parasitol. 2003;33:1561-75

9. Buck AH, Coakley G, Simbari F, Mcsorley H, Quintana J, Le BT, et al. Exosomes secreted by nematode parasites transfer small RNAs to mammalian cells and modulate innate immunity. Nat Commun. 2014;5:5488

10. Knopp S, Steinmann P, Hatz C, Keiser J, Utzinger J. Nematode infections: filariases. Infect Dis Clin North Am. 2012;26(2):359-81.

11. Hotez PJ, Alvarado M, Basáñez M-G, Bolliger I, Bourne R, Boussinesq M, et al. The global burden of disease study 2010: interpretation and implications for the neglected tropical diseases. PLoS Negl Trop Dis. 2014;8:e2865.

12. Morales-Hojas R, Cheke RA, Post RJ. Molecular systematics of five Onchocerca species (Nematoda: Filarioidea) including the human parasite, $\mathrm{O}$. volvulus, suggest sympatric speciation. J Helminthol. 2006;80:281-90.

13. Crump A, Morel CM, Omura S. The onchocerciasis chronicle: from the beginning to the end? Trends Parasitol. 2012;28:280-8.

14. Nfon CK, Makepeace BL, Njongmeta LM, Tanya VN, Bain O, Trees AJ. Eosinophils contribute to killing of adult Onchocerca ochengi within onchocercomata following elimination of Wolbachia. Microbes Infect. 2006:8:2698-705.

15. Wahl G, Enyong P, Ngosso A, Schibel JM, Moyou R, Tubbesing H, et al. Onchocerca ochengi: epidemiological evidence of cross-protection against Onchocerca volvulus in man. Parasitology. 1998;116(Pt 4):349-62.

16. Osei-Atweneboana MY, Eng JKL, Boakye DA, Gyapong JO, Prichard RK. Prevalence and intensity of Onchocerca volvulus infection and efficacy of ivermectin in endemic communities in Ghana: a two-phase epidemiological study. Lancet. 2007;369:2021-9.

17. Gardon J, Gardon-Wendel N, Demanga-Ngangue, Kamgno J, Chippaux JP, Boussinesq M: Serious reactions after mass treatment of onchocerciasis with ivermectin in an area endemic for Loa loa infection. Lancet. 1997; 350:18-22.

18. Boatin BA, Toé L, Alley ES, Nagelkerke NJD, Borsboom G, Habbema JDF. Detection of Onchocerca volvulus infection in low prevalence areas: a comparison of three diagnostic methods. Parasitology. 2002;125(Pt 6):545-52.

19. Park J, Dickerson TJ, Janda KD. Major sperm protein as a diagnostic antigen for onchocerciasis. Bioorg Med Chem. 2008;16:7206-9.

20. Weil GJ, Steel C, Liftis F, Li B, Mearns G, Lobos E, et al. A rapid-format antibody card test for diagnosis of onchocerciasis. J Infect Dis. 2000;186:1796-9.

21. Taniuchi M, Verweij JJ, Noor Z, Sobuz SU, Van Lieshout L, Petri Jr WA, et al. High throughput multiplex PCR and probe-based detection with luminex beads for seven intestinal parasites. Am J Trop Med Hyg. 2011;84:332-7.

22. Srivastava $P$, Mehrotra S, Tiwary P, Chakravarty J, Sundar S. Diagnosis of Indian visceral leishmaniasis by nucleic acid detection using PCR. PLoS One. 2011;6:4-8.

23. Hoy AM, Buck AH. Extracellular small RNAs: what, where, why? Biochem Soc Trans. 2012:40:886-90.

24. Wahl G, Achu-Kwi MD, Mbah D, Dawa O, Renz A. Bovine onchocerciasis in North Cameroon. Vet Parasitol. 1994:52:297-311.

25. Arndts K, Specht S, Debrah AY, Tamarozzi F, Klarmann Schulz U, Mand S, et al. Immunoepidemiological profiling of onchocerciasis patients reveals associations with microfilaria loads and ivermectin intake on both individual and community levels. PLoS Negl Trop Dis. 2014;8:e2679.

26. Burgos KL, Javaherian A, Bomprezzi R, Ghaffari L, Rhodes S, Courtright A, et al. Identification of extracellular miRNA in human cerebrospinal fluid by next-generation sequencing. RNA. 2013;19:712-22. 
27. Martin M. Cutadapt removes adapter sequences from high-throughput sequencing reads. Bioinformatics Act. 2011;17:10-2.

28. Langmead B, Trapnell C, Pop M, Salzberg SL. Ultrafast and memory-efficient alignment of short DNA sequences to the human genome. Genome Biol. 2009;10:R25.

29. Griffiths-Jones S. Rfam: an RNA family database. Nucleic Acids Res. 2003;31:439-41.

30. Friedländer MR, Mackowiak SD, Li N, Chen W, Rajewsky N. miRDeep2 accurately identifies known and hundreds of novel microRNA genes in seven animal clades. Nucleic Acids Res. 2012;40:37-52.

31. Kozomara A, Griffiths-Jones S. miRBase: annotating high confidence microRNAs using deep sequencing data. Nucleic Acids Res. 2014;42(Database issue):D68-73.

32. Gruber AR, Lorenz R, Bernhart SH, Neuböck R, Hofacker IL. The Vienna RNA websuite. Nucleic Acids Res. 2008;36(Web Server issue):W70-4

33. Altschul SF, Gish W, Miller W, Myers EW, Lipman D. Basic Local Alignment Search Tool.pdf. J Mol Biol. 1990;215:403-10.

34. Dhahbi JM, Spindler SR, Atamna H, Yamakawa A, Boffelli D, Mote P, et al. 5' tRNA halves are present as abundant complexes in serum, concentrated in blood cells, and modulated by aging and calorie restriction. BMC Genomics. 2013;14:298.

35. George GH, Palmieri JR, Connor DH. The onchocercal nodule: interrelationship of adult worms and blood vessels. Am J Trop Med Hyg. 1985:34:1144-8.

36. Zouré HGM, Noma M, Tekle AH, Amazigo UV, Diggle PJ, Giorgi E, et al. The geographic distribution of onchocerciasis in the 20 participating countries of the African Programme for Onchocerciasis Control: (2) pre-control endemicity levels and estimated number infected. Parasit Vectors. 2014;7:326.

37. Turner HC, Osei-Atweneboana MY, Walker M, Tettevi EJ, Churcher TS, Asiedu $\mathrm{O}$, et al. The cost of annual versus biannual community-directed treatment of onchocerciasis with ivermectin: Ghana as a case study. PLoS Negl Trop Dis. 2013;7:e2452.

38. Wang $K$, Li H, Yuan Y, Etheridge A, Zhou Y, Huang D, et al. The complex exogenous RNA spectra in human plasma: an interface with human gut biota? PLoS One. 2012;7:e51009.

39. Beatty M, Guduric-fuchs J, Brown E, Bridgett S, Chakravarthy U, Hogg RE, et al. Small RNAs from plants, bacteria and fungi within the order Hypocreales are ubiquitous in human plasma. BMC Genomics. 2014;15:933.

40. Landgraf P, Rusu M, Sheridan R, Sewer A, lovino N, Aravin A, et al. A mammlian microRNA expression atlas based on small RNA library sequencing. Cell. 2007;129:1401-14.

41. Tritten L, Neill MO, Wanji S, Njouendoui A, Fombad F, Kengne-ouaffo J, et al. Loa loa and Onchocerca ochengi miRNAs detected in host circulation. Mol Biochem Parasitol. 2014;198(1):14-7.

42. Shaw WR, Armisen J, Lehrbach NJ, Miska EA. The conserved miR-51 microRNA family is redundantly required for embryonic development and pharynx attachment in Caenorhabditis elegans. Genetics. 2010;185:897-905.

43. Brenner JL, Kemp BJ, Abbott AL. The mir-51 family of microRNAs functions in diverse regulatory pathways in Caenorhabditis elegans. PLOS One. 2012; $7: e 37185$

44. Van der Werf MJ, de Vlas SJ, Brooker S, Looman CW, Nagelkerke NJ, Habbema JDF, et al. Quantification of clinical morbidity associated with schistosome infection in sub-Saharan Africa. Acta Trop. 2003;86:125-39.

\section{Submit your next manuscript to BioMed Central and take full advantage of:}

- Convenient online submission

- Thorough peer review

- No space constraints or color figure charges

- Immediate publication on acceptance

- Inclusion in PubMed, CAS, Scopus and Google Scholar

- Research which is freely available for redistribution 